Sept. 18 . Sehr feine Schattenlinie sichtbar. Blendend weiße Äquatorzonen $z$ wischen $+10^{\circ}$ und $-10^{\circ}$ (kronozentrische Breite).

Sept. r $9.8^{\mathrm{h}} 50^{\mathrm{m}}$. Titan ist schon aus der Scheibe ausgetreten; der Trabant bewegt sich in etwa 2" Abstand nördlich am Ring entlang. $U m I^{h} I^{m}$ ișt er noch etwa $4^{\prime \prime}$ vom Ende der westlichen Anse entfernt; der Austritt aus der Scheibe dürte schon um $8^{\mathrm{h}} 20^{\mathrm{m}}$ stattgefunden haben. Die Tethys, welche um $9^{\mathrm{h}} \mathrm{O}^{\mathrm{m}}$. noch ungefähr $5^{\mathrm{h}}$ westlich vom Ring stand, war $9^{\mathrm{h}} 5^{\mathrm{o}^{\mathrm{m}}}$ verschwunden. Weiße Äquatorzonen wie gestern. Vergr. 243. deutlich.

Okt. I und 2. Ring schwach und schmal. Schatten

Okt. 4. $9^{\text {h }}$. Luft gut. Vergi. 243. Östlich vom Saturn ist eine feine Linie bis etwa $5^{\prime \prime}$ von der Scheibe sichtbar. Die Beobachtung wurde von Herrn $v . d$. Bilt bestätigt. Um $9^{\mathrm{h}} 30^{\mathrm{nt}}$ scheint es dem Amanuensis Kress so, als ob ein sehr schwacher Trabant in einem 4!sian's von $3^{\prime \prime}$ oder 4 " östlich von der Scheibe stehe. Da Rhea nach den Angaben des N. A. schon seit $8^{\text {h }}$ ihren Vorübergang vor der Scheibe angetreten hatte und die anderen Trabanten nicht in Betracht kommen, so glaube ich das Verschwinden des Ringes für das hiesige Instrument auf $10^{\text {h }} \mathrm{cder}$ I $x^{\text {h }}$ festsetzen zu können.

Nov. 2 2. Ring sichtbar. Westliche Anse deutlicher als die östliche.

Nov. 27. Ring deutlich. Diesnual ist die östliche Anse die besser sichtbare.

Nov. 30. Vergr. r64. Beide Ansen gleich hèll.

Dez. 3. Ring viel schwächer; am besten mit Vergr. 94 oder 164 . Luft gut.

Dez. ro. Ring wieder sehr deullich. Die östliche Anse scheint die hellere zu sein; auch $y$. d. Bilt bekam diesen Eindruck.

Dez. r6. Ring schwach: am besten mit Vergr. 94.

Dez. 25. $8^{\mathrm{h}} \circ^{\mathrm{m}}$. Ring schimmert noch schwach (Vergrößerung 94).

I 908 Jan. 4. Ring unsichtbar. Luft gut.

Jan. 5. Ring unsichtbar. Luft seht gut. Ein ziemiich scharf begrenzter grauer Gürtel läuft in der stidlichen Hemisphäre dem Äquator parallel.

Jan. 10. Ring sehr deutlich. Vielleicht schon als Ellipse zu sehen?

Die Messungen des PW. warden mittels eines Doppelfadens von etwa 6" Distanz bei Yergrößerung 243 angesteilt. Bei der großen Schwierigkeit der Messung und dem erheblichen Betrage des zu erwartencien mittleren Fehlers schien

Utrecht, r 908 Febr. 9. es mir unzweckmäßig, die Schraube der Feinbewegung zu benuitzen; ich stellte den Doppelfaden einfach mit der Hand der Ringachse parallel, Das Abendresultat ist das Mittel aus fünf Einstellungen. Für den $m$. F. des Mittels fand ich $0: 16$; für den m. F. einer Einstellung $\circ_{3}^{\circ}$. Da die große Achse des Ringes $45^{\prime \prime}$ betrug, so entspricht dieser $\mathrm{m}$. F. einer linearen Abweichung von o".27. Es wurde jedesmal unmittelbar vach der Messung auf Saturn selbst oder auf Titan der scheinbare Nullpunkt des Positionskreises bestimmt. Bei der geringen Neigung $\left(4^{\circ}\right)$ gegen den Parallelkreis schien es mir überflüssig, die Refraktion zu berücksichtigen. Wenn möglich wurden die Beobachtungen in kleinen Stundenwinkeln angestellt, so daß die Haltung des Kopfes keine Schwierigkeiten bot. Hier folgen die Beobachtungen; die Kolumne B-R enthält den Unterschied des gemessenen PW. gegen die Angabe des Nautical Almanac.

\begin{tabular}{|c|c|c|c|c|c|}
\hline 1907 & M. Z. Utr. & PW. & N. A. & $B-R$ & Luft \\
\hline Juli 30 & $15^{\mathrm{h}} 17^{\mathrm{m}}$ & $3^{\circ} 5^{8}$ & $4: 37$ & $-0: 7.9$ & \\
\hline $3^{1}$ & I 445 & 3.53 & $4 \cdot 37$ & -0.84 & \\
\hline Aug. 4 & 1455 & 4.00 & 4.39 & -0.39 & sehr $g$ \\
\hline 6 & I 342 & 3.68 & 4.39 & -0.71 & \\
\hline I I & 1354 & 3.98 & $4 \cdot 4 x$ & -0.43 & $g u_{t}^{t}$ \\
\hline 27 & 1316 & 3.9 & 4.49 & -0.54 & \\
\hline Sept. 9 & I 348 & 4.77 & 4.57 & +0.20 & \\
\hline II & 1445 & 4.15 & $4 \cdot 5^{8}$ & -0.43 & leidlich \\
\hline 15 & I 3 I 5 & 4.22 & $4.6 \mathrm{I}$ & -0.39 & \\
\hline 19 & I I 0 & 4.40 & 4.63 & -0.23 & \\
\hline
\end{tabular}

Das Mittel der $\mathrm{B}-\mathrm{R}$ ist -0.45 (m. F. \pm 0.096 ).

Auch Herr $v$.d. Bilt hat den PW. öfters gemessen; der m. F. seines Abendresultats ist $0^{\circ} \mathrm{x}$. Hier folgen die Beobachtungen :

\begin{tabular}{|c|c|c|c|c|c|}
\hline 1907 & M.Z. Utr. & PW. & N. A & $B-R$ & Luft \\
\hline Aug. I I & $14^{\mathrm{h}} 10^{\mathrm{m}}$ & $3^{\circ} \cdot 78$ & $4: 4 \mathrm{I}$ & $-0: 63$ & gut \\
\hline 19 & I 350 & 3.37 & 4.45 & -1.08 & unruhig \\
\hline 27 & 1435 & $4 \cdot 5^{8}$ & 4.49 & +0.08 & \\
\hline Sept. 9 & 1238 & 3.98 & 4.57 & -0.59 & sehr gut \\
\hline II & 1340 & 4.98 & $4.5^{8}$ & +0.40 & leidlich \\
\hline 20 & I 45 & 3.97 & 4.64 & -0.67 & \\
\hline 23 & 1255 & 488 & 4.66 & +0.22 & unruhig \\
\hline 26 & 1237 & 4.37 & 4.67 & +0.20 & sehr unruhig \\
\hline
\end{tabular}

Das Mittel der $B-R$ ist -0.26 (m. F. $=0$ ! 19).

$$
\text { A. A. Nijlana. }
$$

\title{
Observations de l'anneau de Saturne en 1907.
}

Jusqu'au 28 juillet nous n'avons pas pu voir l'apparition de l'anneau à l'équatorial de 12 pouces Engelhardt, la clarté de la lune étant très gênante aux observations, mais le trait noir sur le disque était distinctement visible. Le 28 juillet M. Gratschof a pu distinguer l'anneau comme une ligne lumineuse. Moi je ne l'ai vu distinctement que le lendemain. A l'époque de la disparition de l'anneau en octobre, le ciel immédiatement avant le 4 octobre n'était pas clair. Le 4 octobre on ne pouvait voir assurément l'anneau ni à l'observatoire Engelhardt à l'equatorial de I 2 pouces, ni à l'observatoire de Kasan au réfracteur de ro pouces. Mais le $7,8,9$, ro et $\mathbf{I} 7$ novembre l'anneau a été vu à l'équatorial de i 2 pouces par M. Gratschof, qui a fait le 17 nov., $8^{\mathrm{h}}$ t. $\mathrm{m}$. Engelh. les déterminations suivantes: 


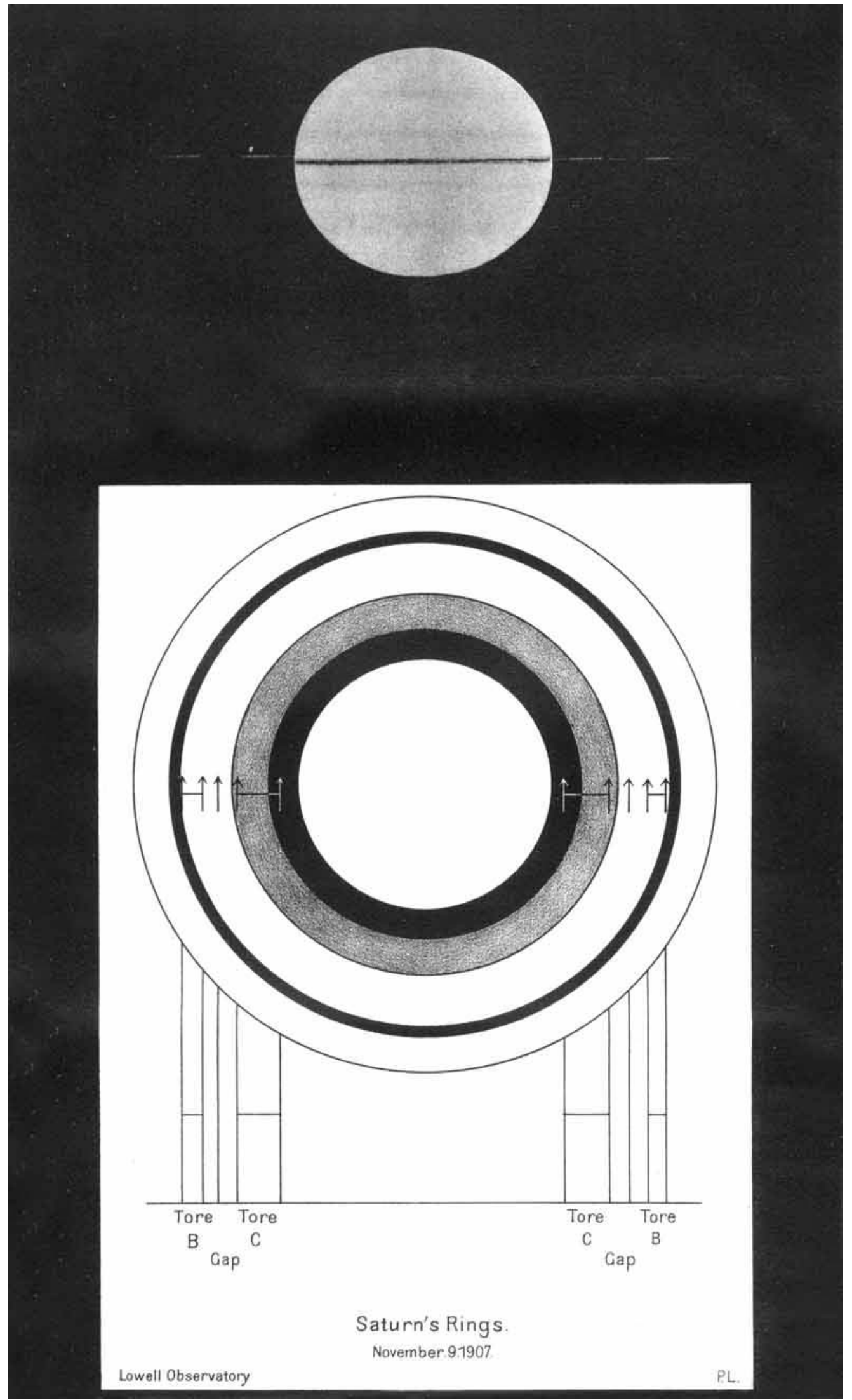


Angle de position de la grande axe $=93^{\circ} 5^{\circ}$ (ro point.)

Diamètre de l'anneau extérieur $=42.27$ ( 5 point.).

M. Baranof et moi, nous avons aussi observé, pendant janvier Igo8 ne pouvait pas être constaté, parce qu'après le mois de novembre, plusieurs fois les traces de l'anneau le I janvier la première nuit claire était celle du $\mathbf{3} 3$ janvier, jusqu'au 26 novembre, après quoi survint un temps brumeux. quand, d'après M. Gratschof, l'anneau était déjà très luLe temps de la nouvelle réapparition de l'anneau en . mineux.

Kasan, le 23 janvier I 908.

D. Doubiago.

Uber den Lichtwechsel von RV Tauri.

Von S. Enebo.

Die definitive Bearbeitung meiner Beobachtungen von RV Tauri hat zu einem höchst interessanten Resultat geführt, das ich im folgenden mitzuteilen mir erlaube.

Es liegen vom Zeitraum 1906 Aug. 27 bis jetzt etwa r oo Beobachtungen vor, deren Ergebnisse in Fig. I graphisch dargestellt sind. Obgleich nun die Punkte besonders bei den Minima zerstreut vorkommen, scheint doch aus der durch die Punkte roh hindurch gezogenen Kurve hervorzugehen, daß erstens der Stern dem $\beta$ Lyrae-Typus angehört und daß zweitens noch eine Nebenveränderlichkeit vorhanden ișt, die aus den höchst verschiedenen Helligkeiten der Maxima am besten hervorgeht.

Um erstens die $\beta$ Lyrae-Veränderlichkeit sicher darzutun, suche ich eine genäherte Periode der tiefsten Minima. Meine Beobachtungen geben nun einen Wert (78.5), der als so genähert angesehen werden kann, dab die von Ceraski in A. N. 4010 mitgeteilten photographischen Daten benutzt werden können, um einen sichereren Periodenwert zu erhalten. Es ergibt sich dann folgende Formel für die Momente der tiefsten Minima:

Min. $=2417609.6$ M.E.Z. $+78.57 E$.
Um danach eine Normalkurve zu erhalten, reduziere ich erstens sämtliche Helligkeiten auf ein System, in welchem ich die Maximalgröße als konstant, gleich der mittleren beobachteten Maximalgröße 9 mo annehme. Alsdann reduziere ich die so erhaltenen einzelnen Helligkeiten auf dieselbe Epoche mit der Periode 78.57 weiter und vereinige sie in Normalpunkte. Das so gewonnene Resultat ist in Fig. 2 dargestellt.

Die Normalkurve scheint nun die vermutete $\beta$ LyraeVeränderlichkeit völlig $z u$ bestätigen, und es können für diese Veränderlichkeit folgende Elemente aufgestellt werden:

$$
\begin{aligned}
\text { Haupt-Min. }=2417609.6 \text { M. E. Z. } & +78.57 E \\
\text { Neben-Min. }=2417648.9 & \$ \quad+78.57 E \\
\text { Max. }=2417629.2 & +39.285 E .
\end{aligned}
$$

Nach den Beobachtungen r 908 Febr. 28 und 29, März I und 3 ist das Licht beim Haupt-Minimum etwa 5 Tage stationär.

Die Nebenveränderlichkeit ist nur für die meine Beobachtungen umfassende Zeit bekannt, weshalb die zuerst erwähnte Reduktion für die photographischen Daten nicht vorgenommen werden kann. $\mathrm{Da}$ es indessen wünschenswert

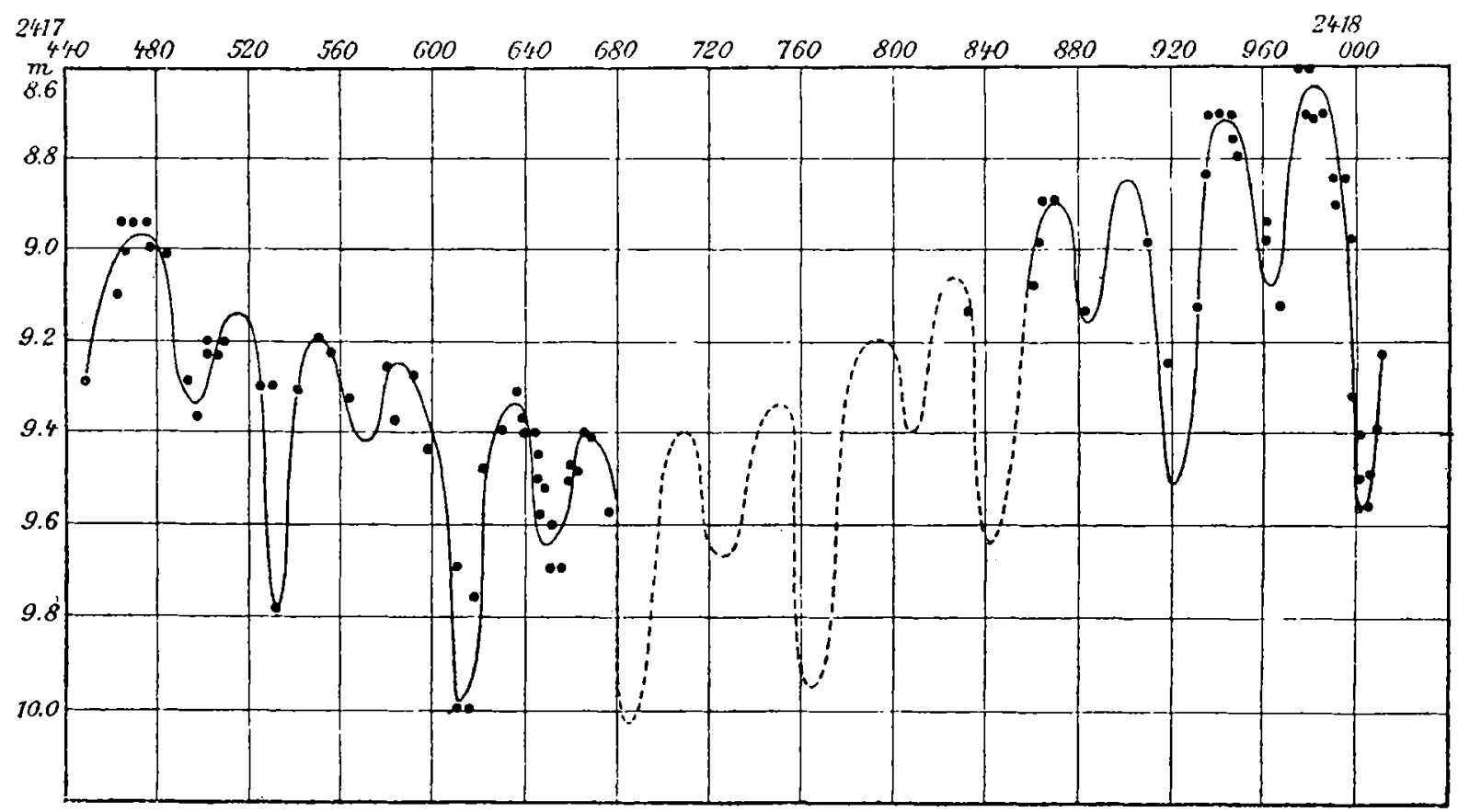

Fig. I. 